Int. J. Electrochem. Sci., 1(2006)1-11

International Journal of

ELECTROCHEMICAL

SCIENCE

www.electrochemsci.org

\title{
Modifed Thomas Algorithm for the Digital Simulation of the Catalytic EC' Mechanism Under Cottrellian Conditions
}

D. Britz

Department of Chemistry, Aarhus University, Langelandsgade 140, 8000 Århus C, Denmark E-mail: britz@chem.au.dk

Received: 3 April 2006 / Accepted: 6 April 2006 / Published: 7 April 2006

An adaptation of the Thomas algorithm is described, that is an alternative to the Rudolph blockThomas algorithm, not using the block vector-matrix technique. This can be used specifically for the classic example of a catalytic $\mathrm{EC}^{\prime}$ mechanism if the regeneration reaction is irreversible.

Keywords: Computational Electrochemistry; Digital Simulation; EC' reaction.

\section{INTRODUCTION AND THEORY}

Until 1991, the simulation of coupled systems, in which electrochemical reactions are coupled with homogeneous chemical reactions in such a way that at least some of the relevant partial differential transport equations ( $p d e s$ ) contain terms for more than one species, was considered hard. The reason is that many such reaction mechanisms lead to thin reaction layers [1], necessitating the use of very small spatial intervals near the electrode. This brings with it the need for implicit methods of solution, and so the discrete system of equations generated from the $p d e s$ became a challenge to solve. Details of this problem can be read in [2]. It is best to present an example of such a system, before describing a solution to the problem. This system is the application of a Cottrellian potential jump to the catalytic reaction pair

$$
\begin{aligned}
& \mathrm{A}+\mathrm{e} \rightarrow \mathrm{B} \\
& \mathrm{B} \rightarrow \mathrm{A}
\end{aligned}
$$

This system is of interest in that it is an example of a coupled system, and an analytical solution was developed by both Delahay and Stiehl [3] and Miller [4] in the same year (1952). The system is thus a good example for testing simulation techniques. The two pdes governing the system are 


$$
\begin{aligned}
& \frac{\partial c_{A}}{\partial t}=D \frac{\partial^{2} c_{A}}{\partial x^{2}}+k c_{B} \\
& \frac{\partial c_{B}}{\partial t}=D \frac{\partial^{2} c_{B}}{\partial x^{2}}-k c_{B}
\end{aligned}
$$

where $c_{X}$ is the concentration of species $\mathrm{X}$ (that is, A or B), $t$ is the time, $D$ is the common diffusion coefficient (assumed here for convenience, although the two species can have different diffusion coefficients and the analytical solutions allow this), $x$ is the spatial dimension and $k$ the forward rate constant of the homogeneous chemical reaction, which is considered irreversible.

Some normalisation is convenient. Initially, species A is assumed to be present at concentration $c_{A}^{*}$, and that of species B to be zero. Let the observation time be $\tau$. Then,

$$
\begin{aligned}
& C_{X}=c_{X} / c_{A}^{*} \\
& T=t / \tau \\
& X=x / \sqrt{D \tau} \\
& K=k \tau
\end{aligned}
$$

This leads to the pair of normalised equations

$$
\begin{aligned}
& \frac{\partial C_{A}}{\partial T}=\frac{\partial^{2} C_{A}}{\partial X^{2}}+K C_{B} \\
& \frac{\partial C_{B}}{\partial T}=\frac{\partial^{2} C_{B}}{\partial X^{2}}-K C_{B}
\end{aligned}
$$

For the Cottrellian potential jump experiment, boundary conditions then are

$$
\begin{array}{ll}
T<0: \quad C_{A}=1, \quad C_{B}=0, \quad \text { all } X \\
T \geq 0: & \\
& C_{A}(X=0)=0, \quad C_{A}(X \rightarrow \infty)=1 \\
& C_{B}(X \rightarrow \infty)=0 \\
& \frac{\partial C_{A}}{\partial X}=-\frac{\partial C_{B}}{\partial X},
\end{array}
$$

The analytical solution for the dimensionless current is then $[3,4]$

$$
I(T)=\sqrt{K} \operatorname{erf} \sqrt{K T}+\frac{\exp (-K T)}{\sqrt{\pi T}}
$$


Unequal spatial intervals must be used because of the reaction layer, and any convenient spacing sequence can be used, such as Feldberg's exponentially expanding sequence [5], giving a sequence of $X$ positions $X_{0}, X_{1}, \ldots, X_{N}, X_{N+1}$, where $X_{N}$ is the maximum distance at which there can be significant diffusional changes; in the dimensionless units used, this is equal to $6 \sqrt{T_{\max }}$ [2], with $T_{\max }$ being the maximum time simulated to. The point at $X_{N+1}$ then lies in the bulk, where the initial bulk concentrations hold throughout. The exponentially expanding sequence of intervals $H$ is given by

$$
H_{i}=\gamma H_{i-1}
$$

giving

$$
X_{i}=X_{1} \frac{\gamma^{i}-1}{\gamma-1}
$$

(Feldberg expresses it differently, in terms of positions of box ends, but point positions are used here); $\gamma$ is the expansion factor, which normally lies between unity and 2. Other sequences can be used but this one is convenient. It is normally generated on the basis of a desired value of $X_{1}$, the position of the point nearest to the electrode, and the desired number of internal points $N$. An algorithm for evaluating $\gamma$, given these two parameters, has been described [2, p.302]. A Fortran 95 function EE_FAC has been written for the calculation.

The spatial second derivative discretisation is performed directly on the unequal grid. Several authors have produced formulae for this $[2,7,8]$, and a general algorithm has been described [6] which can deliver the coeffcients as well as derivative values for first or second derivatives on a number of points, located at any of them. Here, we need the form $u_{2}^{\prime \prime}(3)$, that is, the second derivative located at the second of three points. In the present author's terminology, at the point $X_{i}$,

$$
\frac{\partial^{2} C}{\partial X^{2}} \approx \alpha_{1}(i) C_{i-1}+\alpha_{2}(i) C_{i}+\alpha_{3}(i) C_{i+1}
$$

The $\alpha$ values are dependent on the index $i$. The time derivative discretisation depends on the algorithm to be used in the simulation. Three such are considered here. For the Laasonen method [9], also called BI (for backward implicit) and the Crank-Nicolson method [10], the same discrete expression results:

$$
\frac{\partial C}{\partial T} \approx \frac{C^{\prime}-C}{\delta T}
$$

where $C^{\prime}$ is the new value of concentration to be calculated at time $T+\delta T, \delta T$ being the time interval. If $\mathrm{BDF}[2,11]$ is to be used, the three-point variant is probably optimal, as it has been shown 
[12] that the simplest starting procedure, in which the present and past concentration rows initially both are set at the initial values, and the values of $T$ corrected at each iteration by subtracting $\delta T / 2$, is about optimum for this method. This rather arbitrary correction procedure has been shown to be mathematically justified $[13,14]$. This makes the method second order with respect to $\delta T$ and using more points for BDF does not increase the order, unless a high-order but inefficient starting procedure is used [12]. So, using the three-point variant, we have

$$
\frac{\partial C}{\partial T} \approx \frac{' C-4 C+3 C^{\prime}}{2 \delta T}
$$

where ' $C$ now represents the (known) value at $T-\delta T$.

Using the above discretisations and rearranging the resulting equations, we arrive at the general pair of discrete equations at point $i$ for all three implicit methods

$$
\begin{aligned}
& C_{A, i-1}^{\prime}+p_{i} C_{A, i}+q_{i} C_{B, i}^{\prime}+r_{i} C_{A, i+1}^{\prime}=b_{A, i} \\
& C_{B, i-1}^{\prime}+s_{i} C_{B, i}^{\prime}+r_{i} C_{B, i+1}^{\prime}=b_{B, i}
\end{aligned}
$$

with the coefficients on the left-hand side and the $b$-terms on the right-hand sides given in Table 1 . Because of the coupled nature of (12), the system, and others like it, appeared intractable, unless very long computing times were used. In the Rudolph method, the two equations are gathered into a single one in terms of vectors and matrices, resulting in a block-tridiagonal system. This was introduced to electrochemical simulations by Rudolph [15]. Others had in fact used the technique earlier [16, 17], but it became widely known only after Rudolph's paper. Recently, Bieniasz described a block-Thomas adaptation for a more complex system that is mostly blocktridiagonal but with isolated pentadiagonal rows [18]. For a description of Rudolph's method, see [15] or a recent simulation text [2]. It involves inversion of a number of matrices and matrix-vector multiplications, and the method has the useful feature of being generalisable for general mechanisms with an arbitrary number of species.

For the particular mechanism under discussion here, there is a simpler method, based on a modification of the Thomas algorithm for uncoupled systems. The usual citation for the Thomas algorithm is the book by Thomas [19], but this seems inaccessible now; a description by Bruce et al. [20] may be the earliest accessible description of the standard Thomas algorithm, see also [2]. It will here be combined with another trick, for handling boundary conditions, called the " $u-v$ mechanism" by the present author, and mentioned in previous papers [21,22]. The aim of this paper is to detail this procedure, which avoids vector-matrix manipulation.

Consider the equation pair (12) at the point $i=N$ :

$$
\begin{aligned}
& C_{A, N-1}^{\prime}+p_{N} C_{A, N}^{\prime}+q_{N} C_{B, N}^{\prime}+r_{N} C_{A, N+1}^{\prime}=b_{A, N} \\
& C_{B, N-1}^{\prime}+s_{N} C_{B, N}^{\prime}+r_{N} C_{B, N+1}^{\prime}=b_{B, N}
\end{aligned}
$$


Table 1: Table of coefficients and right-hand sides for the methods BI (Laasonen), Crank-Nicolson (CN) and 3-point BDF.

\begin{tabular}{|l|l|l|l|}
\hline Coeff & $\mathrm{BI}$ & $\mathrm{CN}$ & $\mathrm{BDF}$ \\
\hline$p_{i}$ & $\left(\alpha_{2}(i)-\frac{1}{\delta T}\right) / \alpha_{1}(i)$ & $\left(\alpha_{2}(i)-\frac{2}{\delta T}\right) / \alpha_{1}(i)$ & $\left(\alpha_{2}(i)-\frac{3}{2 \delta T}\right) / \alpha_{1}(i)$ \\
\hline$q_{i}$ & $K / \alpha_{1}(i)$ & $K / \alpha_{1}(i)$ & $K / \alpha_{1}(i)$ \\
\hline$r_{i}$ & $\alpha_{3}(i) / \alpha_{1}(i)$ & $\alpha_{3}(i) / \alpha_{1}(i)$ & $\alpha_{3}(i) / \alpha_{1}(i)$ \\
\hline$s_{i}$ & $\left(\alpha_{2}(i)-K-\frac{1}{\delta T}\right) / \alpha_{1}(i)$ & $\left(\alpha_{2}(i)-K-\frac{2}{\delta T}\right) / \alpha_{1}(i)$ & $\left(\alpha_{2}(i)-K-\frac{3}{2 \delta T}\right) / \alpha_{1}(i)$ \\
\hline$w_{i}$ & $-1 /\left(\alpha_{1}(i) \delta T\right)$ & $\left(\alpha_{2}(i)+\frac{2}{\delta T}\right) / \alpha_{1}(i)$ & $1 /\left(2 \alpha_{1}(i) \delta T\right)$ \\
\hline$z_{i}$ & $-1 /\left(\alpha_{1}(i) \delta T\right)$ & $\left(\alpha_{2}(i)-K+\frac{2}{\delta T}\right) / \alpha_{1}(i)$ & $1 /\left(2 \alpha_{1}(i) \delta T\right)$ \\
\hline$b_{A, i}$ & $w_{i} C_{A, i}$ & $-\left(C_{A, i-1}+w_{i} C_{A, i}\right.$ & $w_{i}\left(C_{A, i}-4 C_{A, i}\right.$ \\
\hline$b_{B, i}$ & $z_{i} C_{B, i}$ & $\left.+q_{i} C_{B, i}+r_{i} C_{A, i+1}\right)$ & \\
\hline
\end{tabular}

Values at $N+1$ are known bulk values, and we can rewrite the equations in reduced form,

$$
\begin{aligned}
& C_{A, N-1}^{\prime}+p_{N} C_{A, N}^{\prime}+q_{N} C_{B, N}^{\prime}=b_{A, N}-r_{N} C_{A, N+1}^{\prime} . \\
& C_{B, N-1}^{\prime}+s_{N} C_{B, N}^{\prime}=b_{B, N}-r_{N} C_{B, N+1}^{\prime}
\end{aligned} .
$$

We proceed first with species B. Its equation is now written as

$$
C_{B, N-1}^{\prime}+s_{N}^{\prime} C_{B, N}^{\prime}=b_{B, N}^{\prime}
$$

with

$$
s_{N}^{\prime}=s_{N}
$$

and

$$
b_{B, N}^{\prime}=b_{B, N}-r_{N} C_{B, N+1}^{\prime}
$$

Also, we can write from (15)

$$
C_{N}^{\prime}=\left(b_{B, N}-C_{B, N-1}^{\prime}\right) / s_{N}^{\prime}
$$

which we insert in the equation for B at $i=N-1$,

$$
C_{B, N-2}^{\prime}+s_{N-1} C_{B, N-1}^{\prime}+r_{N-1} C_{B, N}^{\prime}=b_{B, N-1}
$$


which, after some gathering of terms, leads to

$$
C_{B, N-2}^{\prime}+s_{N-1}^{\prime} C_{B, N-1}^{\prime}=b_{B, N-1}^{\prime}
$$

with

$$
s_{N-1}^{\prime}=s_{N-1}-\frac{r_{N-1}}{s_{N}^{\prime}}
$$

and

$$
b_{B, N-1}^{\prime}=b_{B, N-1}-\frac{r_{N-1}}{s_{N}^{\prime}} b_{B, N}^{\prime} .
$$

This process is continued backwards, generating equations such as

$$
C_{B, i-1}^{\prime}+s_{i}^{\prime} C_{B, i}^{\prime}=b_{B, i}^{\prime}
$$

with

$$
s_{i}^{\prime}=s_{i}-\frac{r_{i}}{s_{i+1}^{\prime}}
$$

and

$$
b_{B, i}^{\prime}=b_{B, i}-\frac{r_{i}}{s_{i+1}^{\prime}} b_{B, i+1}^{\prime}
$$

The recursive process halts at the equation for $i=1$,

$$
C_{B, 0}^{\prime}+s_{1}^{\prime} C_{B, 1}^{\prime}=b_{B, 1}^{\prime}
$$

Now the $\mathrm{u}-\mathrm{v}$ mechanism is applied. Formally, we write

$$
u_{B, 0}=0 ; \quad v_{B, 0}=1
$$

and we can now write all $C_{B, i}^{\prime}$ in terms of $C_{B, 0}^{\prime}$,

$$
C_{B, i}^{\prime}=u_{B, i}+v_{B, i} C_{B, 0}^{\prime}
$$

with the recursive expressions

$$
u_{B, i}=\frac{1}{s_{i}^{\prime}}\left(b_{B, i}^{\prime}-u_{B, i-1}\right) ; \quad v_{B, i}=-\frac{v_{B, i-1}}{S_{i}^{\prime}} .
$$

We still do not know $C_{B, 0}^{\prime}$ but can substitute it for all $C_{B, i}^{\prime}$ values, as in (28). We now turn to species $\mathrm{A}$, and again start with the equation at $i=N$ : 


$$
C_{A, N-1}^{\prime}+p_{N} C_{A, N}^{\prime}+q_{N} C_{B, N}^{\prime}+r_{N} C_{A, N+1}^{\prime}=b_{A, N}
$$

and again, in similar manner, rewrite it as

$$
C_{A, N-1}^{\prime}+p_{N}^{\prime} C_{A, N}^{\prime}+q_{N}^{\prime} C_{B, 0}^{\prime}=b_{A, N}^{\prime}
$$

(note now the presence of the term for species B), with

$$
\begin{aligned}
& p_{N}^{\prime}=p_{N} \\
& q_{N}^{\prime}=q_{N} v_{B, N}
\end{aligned}
$$

and

$$
b_{A, N}^{\prime}=b_{A, N}-r_{N} C_{A, N+1}^{\prime}-q_{N} u_{B, N}
$$

Note the terms $u_{B, N}$ and $v_{B, N}$, arising from substituting (28) for $i=N$. This process leads to the general reduced equation for $\mathrm{A}$ at index $i$,

$$
C_{A, i-1}^{\prime}+p_{i}^{\prime} C_{A, i}^{\prime}+q_{i}^{\prime} C_{B, 0}^{\prime}=b_{A, i}^{\prime}
$$

with the recursive expressions

$$
\begin{gathered}
p_{i}^{\prime}=p_{i}-\frac{r_{i}}{p_{i+1}^{\prime}}, \\
q_{i}^{\prime}=q_{i} v_{B, i}-r_{i} \frac{q_{i+1}^{\prime}}{p_{i+1}^{\prime}}
\end{gathered}
$$

and

$$
b_{A, i}^{\prime}=b_{A, i}-r_{i} \frac{b_{A, i+1}^{\prime}}{p_{i+1}^{\prime}}-q_{i} u_{B, i}
$$

As with species $\mathrm{B}$, this is developed backwards down to $i=1$, where

$$
C_{A, 0}^{\prime}+p_{1}^{\prime} C_{A, 1}^{\prime}+q_{1}^{\prime} C_{B, 0}^{\prime}=b_{A, 1}^{\prime}
$$

We can now apply the boundary conditions (5), discretising fluxes (concentration gradients) as $n$-point approximations on the unequal grid [2] as the $u_{1}^{\prime}(n-1)$ form 


$$
\frac{\partial C(X=0)}{\partial X} \approx \beta_{0} C_{0}+\beta_{1} C_{1}+\ldots+\beta_{n-1} C_{n-1} .
$$

For this, $u$ and $v$ are needed for A as well. Similar manipulation, as for species B yields the general substitution

$$
C_{A, i}^{\prime}=u_{A, i}+v_{A, i} C_{A, 0}^{\prime}+y_{i} C_{B, 0}^{\prime}
$$

for which the recursive expressions are

$$
\begin{aligned}
& u_{A, i}=\frac{1}{p_{i}^{\prime}}\left(b_{A, i}^{\prime}-u_{A, i-1}\right), \\
& v_{A, i}=-\frac{v_{A, i-1}}{p_{i}^{\prime}}
\end{aligned}
$$

and the new

$$
y_{i}=-\frac{1}{p_{i}^{\prime}}\left(y_{i-1}+q_{i}^{\prime}\right)
$$

starting with

$$
u_{A, 0}=0 ; \quad v_{A, 0}=1 ; \quad y_{0}=0 .
$$

Discretising both boundary conditions that are needed, we have

$$
\begin{aligned}
& C_{A, 0}^{\prime}=0 \\
& \beta_{0}\left(C_{A, 0}^{\prime}+C_{B, 0}^{\prime}\right)+\beta_{1}\left(C_{A, 1}^{\prime}+C_{B, 1}^{\prime}\right)+\ldots+\beta_{n-1}\left(C_{A, n-1}^{\prime}+C_{B, n-1}^{\prime}\right)=0
\end{aligned}
$$

and by substituting for all concentration terms, using (28) and (41), as well as for the known (zero) value of $C_{A, 0}^{\prime}$, we have

$$
\sum_{i=0}^{n-1}\left(u_{A, i}+u_{B, i}\right)+C_{B, 0}^{\prime} \sum_{i=0}^{n-1}\left(v_{B, i}+y_{i}\right)=0
$$

from which $C_{B, 0}^{\prime}$ is calculated. All other concentrations now are readily obtained by recursive use, going forward, of (35) and (23).

Alternatively, the Nernst condition or a Butler-Volmer condition can be applied, both of which lead in an obvious manner to a system of two equations, still readily handled, but in these cases no analytical solutions are available for comparison. 


\section{COMPUTATIONAL}

Programming was in Fortran 95, using the Intel compiler 9.0. At least 14 decimals were specified throughout, and computations were performed on a Personal Computer running Linux. Performance was expressed as the logarithm of the relative error in current:

$$
e=\log _{10}\left|\frac{I_{\text {sim }}-I_{\text {anal }}}{I_{\text {anal }}}\right|
$$

where $I_{s i m}$ is the simulated current, and $I_{\text {anal }}$ the analytical solution (6).

\section{RESULTS AND DISCUSSION}

Both the above treatment, as well as the equivalent Rudolph treatment, have been programmed and results were identical for all three implicit methods considered. Also, computer times were very similar, so the above algorithm cannot be said to significantly reduce computing time. It is, however, the impression of the author, that it is simpler to implement in a program than the Rudolph matrixvector method, where largish arrays of vectors and matrices need to be stored.

Fig. 1. shows some performance curves, in terms of the error $\boldsymbol{e}$ as a function of the number of steps in $T$ up to $T=1$, given a fixed grid of 50 points, starting at $X_{1}=0.01$. BDF was started simply, and all times corrected by $\frac{1}{2} \delta T$, as outlined above.

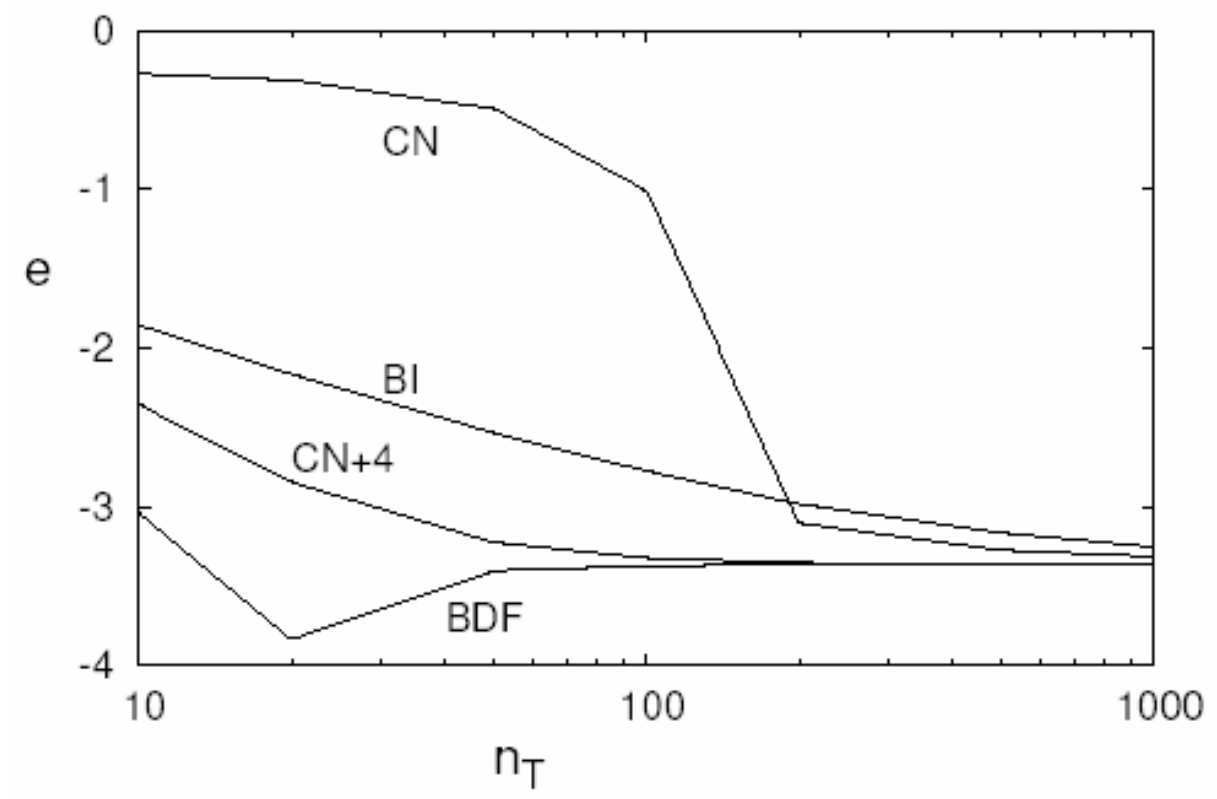

Figure 1: Errors as in equation (48) for the methods BI, CN and BDF as marked. In all but one curve, $N_{X}=50$ and $X_{1}=0.01$, making $\gamma=1.081$. Curve $\mathrm{CN}+4$ was Crank-Nicolson but starting with $4 \mathrm{BI}$ steps to eliminate oscillations. 
It is seen that $\mathrm{BI}$, at least at low values of $N_{T}$, behaves as expected, in a roughly first-order manner, $O(\delta T)$. The BDF curve does behave roughly as $O\left(\delta T^{2}\right)$ as expected, but only at the low end of $N_{T}$, for the first two measured points, but then levels off. $\mathrm{CN}$ does not behave as expected in a second-order manner in any $N_{T}$ range, with or without the BI start. The CN curve clearly shows large errors over most of the range, due to oscillations known from $\mathrm{CN}$. These can be damped by several means [23], and the curve marked as $\mathrm{CN}+4$ shows the result for runs starting with $4 \mathrm{BI}$ steps, clearly an improvement. In fact, this removed the oscillations near the target end time $T=1$ entirely. The ratio $\lambda=\delta T / H^{2}$ plays a crucial role in the oscillations, and for $\lambda<1$ or so, none is seen. In this context, $H$ is the smallest distance from the electrode, here equal to 0.01 , so even for $N_{T}=1000$, we have a $\lambda$ value equal to 10 , still causing some oscillations without the damping start. The higher-order methods are seen to level out to a constant relative log-error of about -3.4, independent of $\delta T$ at higher values of $N_{T}$.

\section{CONCLUSIONS}

The Thomas-like algorithm is seen to produce the same results as the block-tridiagonal Rudolph method, and is simpler to program. The higher-order methods, if suitably implemented, show satisfactory error levels at modest parameter choices. For example, 100 steps in time can bring the relative error down to below $0.1 \%$. Suitable implementation here means the simple start for BDF, subtracting half a time interval at each time or, for $\mathrm{CN}$, starting the simulation with $4 \mathrm{BI}$ steps, to damp oscillations. The algorithm can be used for several boundary conditions, such as Cottrellian as applied here and for which there is an analytical solution, or a Butler-Volmerian or Nernstian condition, for which no analytical solutions are known. Thus, this method can also be used for LSV simulations. This however only applies to the particular mechanism described here, and the method is not general, as the Rudolph method is.

\section{References}

1. K. Wiesner, Chem. Listy 41 (1947) 6.

2. D. Britz, Digital Simulation in Electrochemistry, 3rd Ed., Springer, Heidelberg, 2005.

3. P. Delahay, G. L. Stiehl, J. Am. Chem. Soc. 74 (1952) 3500.

4. S. L. Miller, J. Am. Chem. Soc. 74 (1952) 4130.

5. S. W. Feldberg, J. Electroanal. Chem. 127 (1981) 1.

6. D. Britz, Electrochem. Commun. 5 (2003) 195.

7. D. J. Gavaghan, J. Electroanal. Chem. 456 (1998) 1.

8. M. Rudolph, J. Electroanal. Chem. 529 (2002) 97.

9. P. Laasonen, Acta Math. 81 (1949) 309.

10. J. Crank, P. Nicolson, Proc. Cambridge Phil. Soc. 43 (1947) 50, reprinted in Adv. Comp. Math. 6 (1996) 207, with some slight changes to the list of references.

11. J. Mocak, S. W. Feldberg, J. Electroanal. Chem. 378 (1994) 31.

12. D. Britz, J. Strutwolf, L. Thøgersen, J. Electroanal. Chem. 512 (2001)119.

13. D. Britz, J. Electroanal. Chem. 515 (2001) 1. 
14. T. J. Britz, D. Britz, J. Electroanal. Chem. 546 (2003) 123.

15. M. Rudolph, J. Electroanal. Chem. 314 (1991) 13.

16. J. Newman, Ind. Eng. Chem. Fundam. 7 (1968) 514.

17. R. E. White, Ind. Eng. Chem. Fundam. 17 (1978) 367.

18. L. K. Bieniasz, Computing 67 (2001) 269, erratum: ibid. 70 (2003) 275.

19. L. H. Thomas, Elliptic Problems in Linear Difference Equations over a Network, Watson Scientific Computing Laboratory, Columbia University, New York, 1949.

20. G. H. Bruce, D. W. Peaceman, H. H. Rachford Jr, J. D. Rice, Petr. Trans. AIME 198 (1953) 79.

21. D. Britz, J. Heinze, J. Mortensen, M. Störzbach, J. Electroanal. Chem. 240 (1988) 27.

22. D. Britz, B. M. da Silva, L. A. Avaca, E. R. Gonzales, Anal. Chim. Acta 239 (1990) 87.

23. D. Britz, O. Østerby, J. Strutwolf, Comput. Biol. Chem. 27 (2003) 253.

(C) 2006 by ESG (www.electrochemsci.org) 